\title{
Modelagem da progressão da DBO obtida na incubação de esgoto doméstico sob diferentes temperaturas
}

\author{
Modeling of BOD progression obtained in \\ sewage incubated under different temperatures \\ Mateus Pimentel de Matos ${ }^{1}$, Alisson Carraro Borges ${ }^{2}$, \\ Antonio Teixeira de Matos ${ }^{3}$, Edson Faria da Silva ${ }^{4}$, Mauro Aparecido Martinez ${ }^{5}$
}

口-

\begin{abstract}
RESUMO
Confiável quantificação da demanda bioquímica de oxigênio exercida (DBO exercida) poderá ser obtida com incubação das amostras sob temperaturas diferentes, desde que o valor da demanda bioquímica de oxigênio última $\left(\mathrm{DBO}_{\mathrm{u}}\right)$ não seja alterado nessas condições. Alguns autores têm relatado aumento no valor da $\mathrm{DBO}_{u}$ com a elevação da temperatura de incubação das amostras, o que pode ser decorrente da ocorrência da nitrificação, da aclimatação diferenciada ou do mau ajuste dos modelos de progressão da demanda bioquímica carbonácea de oxigênio (DBO exercida). Neste estudo, objetivou-se avaliar o efeito da temperatura sobre a progressão da DBO exercida e o valor da $\mathrm{DBO}_{u}$ em amostras de esgoto doméstico bruto, avaliando-se qual modelo melhor se ajusta aos dados obtidos. Os resultados indicaram pequena influência da temperatura de incubação das amostras nos valores da $\mathrm{DBO}_{u}$ do esgoto sanitário e que os modelos de primeira e segunda ordens descreveram adequadamente a progressão da DBO exercida.
\end{abstract}

Palavras-chave: demanda bioquímica de oxigênio última; matéria orgânica carbonácea; modelos de progressão da DBO.

\begin{abstract}
Reliable quantification of biochemical oxygen demand exerted (exerted $\mathrm{BOD}$ ) can be obtained by incubating samples at different temperatures, since the amount of ultimate biochemical oxygen demand (UBOD) does not change in these conditions. Some authors have found increased UBOD values with rise in temperature of incubating samples, which could be due to the occurrence of nitrification, the acclimatization or the inadequacy of modeling of carbonaceous oxygen demand (exerted BOD) progression. This study aimed to evaluate the effect of temperature on the progression of exerted BOD and UBOD values in samples of raw domestic sewage, checking which model best fits the data. The results indicated low influence of incubating sewage samples temperature on UBOD values, being that both models, first and second order, described properly the exerted BOD progression.
\end{abstract}

Keywords: carbonaceous organic matter; progression models of $\mathrm{BOD}$; ultimate biochemical oxygen demand.

\section{INTRODUÇÃO}

Em determinadas situações, notadamente quando se requer tomada de decisão operacional, pode ser necessária a quantificação da demanda bioquímica de oxigênio exercida (DBO exercida) em amostras em períodos de tempo menores que cinco dias. Nesse caso, essa redução no tempo de análise pode ser obtida com o aumento da temperatura de incubação das amostras, como foi obtido por Matos et al. (2014). Entretanto, isso só geraria resultados confiáveis se essa alteração na temperatura não viesse a proporcionar interferências na progressão da DBO exercida ao longo do tempo e alterações no valor da demanda bioquímica de oxigênio última $\left(\mathrm{DBO}_{\mathrm{u}}\right)$.

$\mathrm{A} \mathrm{DBO}_{\mathrm{u}}$ equivale à concentração de matéria orgânica biodegradável inicial, ou à quantidade de oxigênio dissolvido $(\mathrm{OD})$ requerido para a total degradação do material orgânico (APHA; AWWA; WEF, 2012). Sua estimativa é importante em estudos de modelagem da qualidade da água em rios, como autodepuração, possibilitando que se efetuem

Doutor pela Universidade Federal de Minas Gerais (UFMG). Professor da Universidade Federal de Lavras (UFLA) - Lavras (MG), Brasil.

²Doutor pela Universidade de São Paulo (USP). Professor da Universidade Federal de Viçosa (UFV) - Viçosa (MG), Brasil.

${ }^{3}$ Doutor pela UFV. Professor da UFMG - Belo Horizonte (MG), Brasil.

${ }^{4}$ Mestre pela UFV. Professor da Faculdade do Noroeste de Minas (FINOM) - Paracatu (MG), Brasil.

${ }^{5}$ Doutor pela Purdue University. Professor da UFV - Viçosa (MG), Brasil.

Endereço para correspondência: Alisson Carraro Borges - Avenida Peter Henry Rolfs, s/n, Departamento de Engenharia Agrícola - Campus Universitário - $36570-900$ - Viçosa (MG), Brasil - E-mail: borges@ufv.com.br

Recebido em: 14/09/12 - Aceito em: 30/08/16 - Reg. ABES: 101993 
estimativas da sua capacidade de recuperação; a verificação do atendimento à legislação, além do seu valor ser referencial para a cinética de desoxigenação no corpo d'água. A taxa com que se alcança a $\mathrm{DBO}_{u}$, ou com que há o consumo de oxigênio ao longo do tempo, está relacionada ao coeficiente de desoxigenação $(\mathrm{k})$.

Tanto o valor de k como a $\mathrm{DBO}_{\mathrm{u}}$ podem ser obtidos como parâmetros ajustados em modelos de progressão na degradação da demanda exclusivamente carbonácea de oxigênio. Dessa forma, quaisquer outros compostos químicos que requeiram OD para sua oxidação, tais como as formas reduzidas de nitrogênio $(\mathrm{N})$, são considerados interferentes e prejudicam tanto o ajuste de modelos aos dados quanto a obtenção de $\mathrm{k}$ e de $\mathrm{DBO}_{\mathrm{u}}$ e as estimativas da real eficiência de tratamento de sistemas biológicos (MAY, 1971).

Diversos são os métodos citados na literatura para a supressão da demanda nitrogenada de oxigênio, dentre eles a acidificação, uma vez que o $\mathrm{pH}$ ótimo para o metabolismo dos microrganismos autotróficos nitrificantes é por volta de 7,5 a 8,0 (TCHOBANOGLOUS; EDDY, 2003); a pasteurização, proporcionando a morte dos microrganismos presentes pela elevação da temperatura; e a adição de substâncias xenobióticas, como o azul de metileno. Essas técnicas são complexas, e sua aplicação exige mão de obra especializada para não prejudicar a análise e obter resultados confiáveis (ALBERTSON, 1995).

Para inibir a nitrificação no meio, APHA, AWWA e WEF (2012) recomendam o uso do 2-cloro-6-triclorometilpiridina (TCMP), na concentração de $3 \mathrm{mg}$ para cada frasco de DBO (aproximadamente $300 \mathrm{~mL}$ ), porém desaconselham seu uso na determinação da DBO se a medição do OD remanescente for feita antes de cinco dias de incubação da amostra sob temperatura de $20^{\circ} \mathrm{C}$, no caso de efluentes brutos e tratados apenas em nível primário. Tal recomendação advém do fato de que não haveria tempo suficiente para o início do processo de nitrificação no meio e, com isso, se incorreria em erros de subestimativa no valor de DBO exercida, tendo em vista a possibilidade da ocorrência de ligações do TCMP aos substratos ou às próprias enzimas das bactérias presentes na amostra (ALBERSTON, 1995); ou superestimativa, em decorrência do consumo de OD para a oxidação da substância anti-nitrificante, aplicada em excesso ou sem necessidade (MUIRHEAD et al., 2006). Assim, o ideal é usar a inibição da nitrificação nos dias em que realmente ocorrer a demanda de segundo estágio, o que é, entretanto, de difícil previsão.

Segundo Dague (1981), as progressões nas demandas carbonácea e nitrogenada se iniciariam quase ao mesmo tempo em efluentes tratados biologicamente, enquanto em efluentes brutos, tal como descrito por Green \& Ward (2011), a nitrificação ocorreria entre o quinto e oitavo dia de incubação das amostras. Esse atraso no começo da nitrificação se deve, principalmente, a dois fatores: primeiro, à própria disponibilização do íon reduzido (amônio) e, segundo, ao desenvolvimento mais lento das bactérias nitrificantes, quando comparadas às bactérias heterotróficas, o que acarreta em atraso na mensuração da demanda nitrogenada (TCHOBANOGLOUS \& EDDY, 2003). De acordo com Dague (1981), as taxas de reprodução das bactérias nitrificantes correspondem a cerca de $10 \%$ daquelas obtidas pelas bactérias heterotróficas (consumidoras de material orgânico).

A ocorrência do processo de nitrificação em uma amostra pode ser identificada na progressão da DBO exercida, quando há mudança na tendência da curva da demanda de primeiro estágio, havendo sobrelevação da mesma. O surgimento de picos de DBO exercida e o mau ajuste aos modelos de progressão carbonácea mais usados, como os de primeira e segunda ordens, também indicam a ocorrência da demanda nitrogenada. Na literatura, há divergência sobre qual das equações pode melhor descrever o consumo de OD e, portanto, a DBO exercida ao longo do tempo. Autores como May (1971) defendem o uso do modelo apresentado na Equação 1, pois a soma das progressões do consumo de substratos simples remeteria a curvas de cinética do tipo de primeira ordem, nas quais $\mathrm{k}$ possui unidade $\left[\mathrm{T}^{-1}\right]$ :

$\mathrm{DBO}_{\mathrm{t}}=\mathrm{DBO}_{\mathrm{u}} \cdot\left(1-\mathrm{e}^{-\mathrm{k.t}}\right)$

Em que:

$\mathrm{DBO}_{\mathrm{t}}$ é a demanda bioquímica de oxigênio exercida em um tempo $\mathrm{t}$ qualquer;

$\mathrm{DBO}_{\mathrm{u}}$ é a demanda bioquímica de oxigênio última; e

k é o coeficiente de desoxigenação e t é o tempo.

Segundo Zanoni (1967) e Adrian \& Sanders (1998), para a descrição da progressão da DBO exercida com o tempo, seria melhor utilizar equações de segunda ordem, pois a soma das progressões do consumo de cada composto presente na amostra seria mais complexa que o modelo de primeira ordem, e a Equação 1 seria utilizada mais por simplicidade do que por embasamento científico. A cinética de segunda ordem para a progressão da DBO exercida em função do tempo pode ser apresentada como na Equação 2:

$\mathrm{DBO}_{\mathrm{t}}=\frac{\mathrm{k}^{\prime \prime} \cdot\left(\mathrm{DBO}_{\mathrm{u}}\right)^{2} \cdot \mathrm{t}}{1+\mathrm{k}^{\prime \prime} \cdot \mathrm{DBO}_{\mathrm{u}} \cdot \mathrm{t}}$

Em que:

k" é o coeficiente de desoxigenação de segunda ordem.

Segundo Adrian \& Sanders (1998), uma das vantagens da utilização do modelo da cinética de segunda ordem é a obtenção de maiores valores de $\mathrm{DBO}_{\mathrm{u}}$, proporcionando maior segurança em estudos de autodepuração - obtendo-se cenário de condições mais críticas. Outros autores recomendam a utilização de modelos de ordem fracionária (BORSUK \& STOW, 2000) ou a obtenção de modelos distintos, separadamente, para cada composto orgânico presente (MASON; 
MCLACHLAN; GÉRARD, 2006). Tal estratégia pode ser considerada muito complexa para a modelagem da progressão desse tipo de variável, que sabidamente apresenta grande desvio padrão.

No modelo de segunda ordem, a $\mathrm{DBO}$ exercida $\left(\mathrm{DBO}_{\mathrm{t}}\right)$ está relacionada à $\mathrm{DBO}_{\mathrm{u}}$ pelo $\mathrm{k}$ ", que tem unidade $\left[\mathrm{L}^{3} \mathrm{~T}^{-1} \mathrm{M}^{-1}\right]$. Para que esse parâmetro possa ser comparado, em termos de magnitude, aos valores de k obtidos com a utilização da Equação 1, mais difundida e utilizada na literatura, deve-se utilizar um artifício. Um coeficiente k', com a mesma unidade de primeira ordem $\left[\mathrm{T}^{-1}\right]$, é estimado pelo produto entre $\mathrm{DBO}_{\mathrm{u}}$ e o k" obtido no ajuste da Equação 2.

Por definição, a $\mathrm{DBO}_{\mathrm{u}}$ não deveria variar com a temperatura, já que é a máxima (potencial) quantidade de OD necessária para degradar todo o material orgânico biodegradável presente na amostra. Assim, não seria de se esperar que a elevação da temperatura viesse a majorar o seu valor, mas apenas a reduzir o período de tempo necessário para que fosse obtido, em função do aumento na taxa de degradação do material orgânico, observável pelo aumento no valor de $\mathrm{k}$. No entanto, alterações no valor da $\mathrm{DBO}_{\mathrm{u}}$ com a temperatura podem estar associadas à combinação entre a cinética de degradação da matéria orgânica diretamente biodegradável e a competição entre microrganismos heterotróficos e nitrificantes por oxigênio, no meio. Com o aumento da temperatura, a conversão de compostos orgânicos será efetuada mais rapidamente, podendo haver, portanto, disponibilidade de oxigênio para os microrganismos nitrificantes, em momentos distintos, a depender da temperatura de incubação da amostra.

$\mathrm{Na}$ Equação 3 está representada a relação de Arrhenius, modificada por Streeter \& Phelps (1925), que correlaciona o k a uma temperatura $\mathrm{T}$ qualquer ao k obtido na temperatura de $20^{\circ} \mathrm{C}\left(\mathrm{k}_{20}\right)$ e ao coeficiente de temperatura $(\theta)$, que é igual a 1,047 .

$\mathrm{k}_{\mathrm{T}}=\mathrm{k}_{20} \cdot \theta^{(\mathrm{T}-20)}$

Contrariando as expectativas, Theriault (1927), Moore (1941) e Zanoni (1967) verificaram aumento no valor da $\mathrm{DBO}_{\mathrm{u}}$ com elevação da temperatura, observando tal acréscimo pelo coeficiente de temperatura da $\mathrm{DBO}_{\mathrm{u}}(\varphi)$ (Equação 4):

$\mathrm{DBO}_{\mathrm{u}}^{\mathrm{T}}=\mathrm{DBO}_{\mathrm{u}}^{20} \cdot[1+\varphi \cdot(\mathrm{T}-20)]$

Em que:

$\mathrm{DBO}_{\mathrm{u}}^{\mathrm{T}}$ é a demanda bioquímica de oxigênio última obtida após incubação da amostra à temperatura $\mathrm{T}$.

Theriault (1927) encontrou valor de $\varphi$ de 0,02 , indicando aumento de $2 \%$ na $\mathrm{DBO}_{\mathrm{u}}$ a cada elevação de $1^{\circ} \mathrm{C}$ na temperatura de incubação da amostra. Moore (1941), avaliando dois efluentes diferentes, um rico em $\mathrm{N}\left(22,5 \mathrm{mg} \mathrm{L}^{-1}\right)$ e outro com concentração menor $\left(11,0 \mathrm{mg} \mathrm{L}^{-1}\right)$, obteve, respectivamente, valores de 0,0260 e 0,0082. Já Zanoni (1967), utilizando o modelo de primeira ordem, encontrou $\varphi$ de 0,0033 , e, usando o modelo de segunda ordem, não verificou diferença considerável entre os valores de $\mathrm{DBO}_{\mathrm{u}}$ em progressões de demanda de oxigênio obtidas em amostras submetidas a diferentes temperaturas de incubação. Diferentemente dos autores citados anteriormente, Gotaas (1948) e May (1971) não observaram variação apreciável nos valores de $\mathrm{DBO}_{u}$ com o aumento na temperatura de incubação das amostras. Utilizando a Equação 4, o primeiro autor obteve valor igual a 0,0013 na faixa de temperatura de 5 a $40^{\circ} \mathrm{C}$-, enquanto o segundo, além de não encontrar variação na $\mathrm{DBO}_{\mathrm{u}}$, ainda apresentou explicações plausíveis para a ocorrência dessas variações nos trabalhos conduzidos por outros pesquisadores. Segundo May (1971), os resultados de Moore (1941) foram influenciados pela nitrificação na amostra, como pode ser verificado pelo baixo coeficiente $\varphi$ encontrado, quando a $\mathrm{DBO}_{u}$ foi obtida em amostras com baixa concentração de N. O valor de $\varphi$ obtido nas amostras contendo altas concentrações de $\mathrm{N}$ foi semelhante ao encontrado no trabalho de Theriault (1927), indicando que, possivelmente, a nitrificação também foi a causadora da diferença entre os valores das $\mathrm{DBO}_{\mathrm{u}}$ obtidas no trabalho desse autor. É sabido que a elevação da temperatura favorece a demanda nitrogenada, sendo que a faixa de temperatura ótima para a ocorrência de nitrificação está entre 25 e $28^{\circ} \mathrm{C}$, segundo Courchaine (1968) e Judkings et al. (1992), os últimos citados por Isoldi, Koetz e Ioldi (2005). Dessa maneira, a elevação da temperatura pode favorecer conclusões errôneas sobre a mudança nos valores de $\mathrm{DBO}_{\mathrm{u}}$, devido ao consumo de oxigênio para a oxidação das formas reduzidas de $\mathrm{N}$.

Ainda segundo May (1971), o possível mau ajuste dos dados de progressão da DBO exercida à equação de primeira ordem, tal como reportado por Zanoni (1967), estaria relacionado ao fato de terem sido utilizadas águas residuárias submetidas ao congelamento antes da realização das análises. Após descongeladas, as amostras eram, ainda, misturadas, aeradas e só depois incubadas, em um procedimento que o próprio autor reconheceu como prejudicial à análise, já que houve $10 \%$ de diferença entre a $\mathrm{DBO}_{\mathrm{u}}^{20}$ obtida no efluente original e aquela obtida no efluente descongelado (ZANONI, 1965). Simon et al. (2011) reportaram que amostras frescas de esgoto sanitário bruto, analisadas em menos de $6 \mathrm{~h}$ após sua coleta, apresentaram valor de $\mathrm{DBO}_{\mathrm{u}}^{20}-$ amostras incubadas por 7 dias a $20^{\circ} \mathrm{C}$ - cerca de $60 \%$ maior do que no efluente bruto, armazenado a $4^{\circ} \mathrm{C}$, no escuro, por um período maior que um dia e menor que um mês. Esses resultados corroboram a hipótese de que a degradação do material orgânico em armazenamentos por longos períodos proporciona subestimativa no valor da DBO exercida em águas residuárias.

Conforme relatado, em alguns estudos foi verificado aumento no valor da $\mathrm{DBO}_{\mathrm{u}}$ com a elevação da temperatura de incubação das amostras, entretanto suspeita-se que isso possa ser decorrente da não 
computação da demanda de oxigênio no processo da nitrificação, da aclimatação diferenciada ou do mau ajuste dos modelos de progressão da DBO exercida. Dessa forma, para que se possa ter confiabilidade nas estimativas de DBO exercida obtidas sob mais altas temperaturas de incubação da amostra, objetivou-se, com a realização deste trabalho, avaliar o efeito da temperatura sobre a progressão da DBO exercida e sobre os valores de $\mathrm{DBO}_{u}$ em amostras de esgoto doméstico bruto (EDB), verificando-se qual modelo de cinética de reação (primeira ou segunda ordens) melhor se ajusta aos dados.

\section{MATERIAL E MÉTODOS}

O trabalho foi conduzido no Laboratório de Qualidade da Água, do Departamento de Engenharia Agrícola, na Universidade Federal de Viçosa e consistiu de análises da DBO em amostras de EDB proveniente de uma residência de quatro moradores do município de Viçosa, Minas Gerais. O EDB, basicamente composto por águas negras, apresentou medianas de 450, 80 e $12 \mathrm{mg} \mathrm{L}^{-1}$ nas concentrações, respectivamente, de $\mathrm{DBO}_{\mathrm{u}}^{20}, \mathrm{~N}$ e fósforo total, obtidas em 2 anos de monitoramento (SILVA et al., 2013).

Para não incorrer em erros, tais como os relatados em APHA, AWWA e WEF (2012) e no trabalho de Simon et al. (2011), que podem proporcionar inclusive mau ajuste do modelo de primeira ordem, tal como ocorreu no trabalho de Zanoni (1967), o tempo entre a coleta e o preparo das amostras para incubação não foi maior do que $6 \mathrm{~h}$. No período em que ficou em espera, a amostra permaneceu refrigerada a $4^{\circ} \mathrm{C}$ para reduzir o risco de degradação do material orgânico durante $o$ armazenamento.

Para o estabelecimento dos volumes de amostra de EDB a serem usados nas análises e a obtenção da progressão da DBO exercida, foi analisada a demanda química de oxigênio (DQO) pelo método do refluxo aberto, procedimento que possibilita ter uma estimativa prévia da concentração de material orgânico na amostra e, consequentemente, da diluição necessária. Os volumes de amostras usados na incubação sob temperatura de $20^{\circ} \mathrm{C}$, por $1,3,5,7$ e 8 dias, foram respectivamente de 10,$0 ; 6,0 ; 3,0 ; 2,0 ; 1,0 \mathrm{~mL}$ e, na incubação sob temperatura de $35^{\circ} \mathrm{C}$, por $1,2,3,5$ e 7 dias, de 8,00; 6,00; 1,00; 0,25 e $0,20 \mathrm{~mL}$. O volume de amostras utilizado foi decrescente em relação ao tempo de incubação, já que a DBO é crescente até se alcançar o valor da $\mathrm{DBO}_{\mathrm{u}}$. A temperatura de incubação das amostras de $\mathrm{DBO}$ a $20^{\circ} \mathrm{C}$ foi escolhida por ser convencionalmente utilizada na sua análise da $\mathrm{DBO}$, e a de $35^{\circ} \mathrm{C}$, por ser o valor encontrado que proporcionou maior taxa de degradação do material orgânico nos trabalhos de May (1971) e Ji et al. (2010), além de ser a temperatura ótima para a ação dos microrganismos em efluentes com maior concentração de material orgânico, conforme verificado por May (1971). Dessa forma, poderia favorecer maior degradação e aumento do consumo de OD, seja pela demanda carbonácea, seja pela nitrogenada, que também é majorada.

O procedimento empregado para a realização da análise da DBO foi o método de Winkler (iodométrico), descrito em APHA, AWWA e WEF (2012). Para minimizar a nitrificação sem a adição de substâncias anti-nitrificantes, como o TCMP, que proporcionaria aumento na demanda por oxigênio, fizeram-se as medições em amostras incubadas sob temperatura de $20^{\circ} \mathrm{C}$, por tempos de 0 (branco), 1, 3, 5, 7 e 8 dias, com 3 repetições. O tempo de oito dias foi considerado o demandado para o início da nitrificação, conforme sugerido por Green \& Ward (2011). De fato, foi observada significativa elevação na demanda de oxigênio na amostra, nas curvas de progressão da DBO exercida após o oitavo dia.

De forma semelhante, amostras foram incubadas sob temperatura de $35^{\circ} \mathrm{C}$, com leituras de OD residual realizadas nos tempos 0 (branco), 1, 2, 3, 5 e 7 dias. Nesse caso, a interrupção das medições antes do oitavo dia se deveu à obtenção de valor de DBO acima do esperado para a demanda carbonácea total. Considerando-se que a progressão da $\mathrm{DBO}$ na amostra submetida à temperatura de incubação de $35^{\circ} \mathrm{C}$ seria aproximadamente duas vezes mais rápida do que a obtida na amostra incubada sob a temperatura de $20^{\circ} \mathrm{C}$ (MATOS et al., 2014), a expectativa era de que a nitrificação também se iniciasse em um tempo mais curto de incubação das amostras. A análise da curva de progressão da DBO exercida indicou evidências nesse sentido, tendo sido observada mudança na sua tendência. Para o cálculo da DBO exercida, foi utilizada a equação apresentada em APHA, AWWA e WEF (2012) e modificada por Matos (2012) (Equação 5):

$\mathrm{DBO}_{\mathrm{t}}=\left\{\frac{\left[\left(\mathrm{OD}_{\mathrm{Br} 0}-\mathrm{OD}_{\mathrm{t}}\right)-\left(\mathrm{OD}_{\mathrm{Br} 0}-\mathrm{OD}_{\mathrm{Brt}}\right)\right] \times\left(\frac{\mathrm{V}_{\mathrm{f}}-\mathrm{V}_{\mathrm{am}}}{\mathrm{V}_{\mathrm{fBr}}}\right)}{\frac{\mathrm{V}_{\mathrm{am}}}{\mathrm{V}_{\mathrm{f}}}}\right\}$

em que,

$\mathrm{DBO}_{\mathrm{t}}$ Demanda bioquímica de oxigênio exercida na amostra em um tempo " $\mathrm{t}$ " ( $\left.\mathrm{mg} \mathrm{L}^{-1}\right)$;

$\mathrm{OD}_{\mathrm{Br} 0}$ Oxigênio dissolvido na amostra "branco", ou seja, no tempo 0 dias $\left(\mathrm{mg} \mathrm{L}^{-1}\right)$;

$\mathrm{OD}_{\mathrm{t}}$ Oxigênio dissolvido na amostra após um tempo $\mathrm{t}$ dias $\left(\mathrm{mg} \mathrm{L}^{-1}\right)$; $\mathrm{OD}_{\text {Brt }}$ Oxigênio dissolvido na amostra "branco" no tempo t dias $\left(\mathrm{mg} \mathrm{L}^{-1}\right)$; $\mathrm{V}_{\mathrm{f}}$ Volume do total de água (amostra + solução nutritiva) no frasco $(\mathrm{mL})$; $\mathrm{V}_{\mathrm{am}}$ Volume de amostra bruta inoculada no frasco especial de DBO $(\mathrm{mL})$; $\mathrm{V}_{\mathrm{fBrt}}$ Volume do frasco da amostra "branco" no tempo t dias (mL).

Essa fórmula foi utilizada porque elimina as depleções na água de diluição, que poderiam ocorrer em caso de contaminação das amostras 
e de diminuição na solubilidade do oxigênio na água submetida a maiores temperaturas de incubação.

Os modelos de primeira e segunda ordens, Equações 1 e 2, respectivamente, foram ajustados aos dados obtidos sob as duas temperaturas de incubação das amostras, utilizando-se a regressão não linear, que, segundo Oke \& Akindahunsi (2005), é o método mais preciso para a estimativa do $\mathrm{k}$ e do valor de $\mathrm{DBO}_{\mathrm{u}}$. Para verificar qual dos dois modelos melhor se ajustou aos dados de DBO, foram avaliados os critérios coeficiente de determinação $\left(R^{2}\right)$ e $R^{2}$ ajustado $\left(\mathrm{R}_{\mathrm{AJ}}^{2}\right)$. Embora os dados de $\mathrm{DBO}$ exercida não apresentem distribuição normal, considerou-se conveniente calcular, também, o erro padrão de estimativa ou raiz quadrada do erro quadrático médio (RMSE) e o critério de informação de Akaike (AIC), que, segundo Emiliano et al. (2009), é um dos métodos mais adequados para a comparação e seleção de modelos, utilizando-se pequenas bases de dados. Esses critérios foram avaliados com a utilização dos aplicativos Sigmaplot 10.0 e Origin 8.0. Gagliasso et al. (2014) também utilizaram o RMSE como critério na escolha do melhor modelo - dentre paramétricos e não paramétricos - para a estimativa da biomassa florestal.

Após a obtenção do k e da $\mathrm{DBO}_{\mathrm{u}}$, pode-se calcular os valores de $\theta$ e de $\varphi$, utilizando-se, respectivamente, as Equações 3 e 4 .

\section{RESULTADOS E DISCUSSÃO}

Na Tabela 1, estão apresentados os valores médios de DBO exercida nas amostras de EDB incubadas sob as temperaturas de 20 e $35^{\circ} \mathrm{C}$. Verificou-se que o valor da DBO exercida nas amostras incubadas sob temperatura de $35^{\circ} \mathrm{C}$, a partir do quinto dia, superou o considerado normal para a $\mathrm{DBO}_{\mathrm{u}}$ de $\mathrm{EDB}$, que é em torno de $438 \mathrm{mg} \mathrm{L}^{-1}$, segundo von Sperling (2014), valor também observado por Silva et al. (2013).

Tabela 1 - Valores médios de demanda bioquímica de oxigênio exercida nas amostras submetidas a diferentes tempos de incubação, sob temperaturas de 20 e $35^{\circ} \mathrm{C}$. Entre parênteses, o desvio padrão da média dos valores de demanda bioquímica de oxigênio.

\begin{tabular}{l|c|c}
\multirow{2}{*}{ Dias } & \multicolumn{2}{|c}{ DBO $\left(\mathrm{mg} \mathrm{L}^{-1}\right)$} \\
\cline { 2 - 3 } & \multicolumn{2}{|c}{ Temperatura } \\
\cline { 2 - 3 } 0 & $20^{\circ} \mathrm{C}$ & $35^{\circ} \mathrm{C}$ \\
\hline 1 & 0 & 0 \\
\hline 2 & $100(5)$ & $172(5)$ \\
\hline 3 & - & $242(3)$ \\
\hline 5 & $240(9)$ & $289(25)$ \\
\hline 7 & $283(17)$ & $462(65)$ \\
\hline 8 & $386(26)$ & $859(236)$ \\
\hline
\end{tabular}

Em negrito, os valores utilizados para obtenção dos coeficientes de desoxigenação. DBO: demanda bioquímica de oxigênio.
Nas condições do experimento, a atividade nitrificante parece ter exercido papel expressivo no consumo de oxigênio do meio líquido a partir do quinto dia de incubação das amostras de EDB, sendo, por essa razão, a concentração de $\mathrm{DBO}_{7}^{35}$ desconsiderada no ajuste aos modelos de progressão da $\mathrm{DBO}$ exercida.

O aparecimento da demanda nitrogenada pode ser evidenciado pelas linhas de tendência da DBO exercida, seguindo o modelo de primeira ordem. Na Figura 1 está apresentada a curva de DBO exercida, plotada com dados observados e ajustados na incubação das amostras sob temperatura de $35^{\circ} \mathrm{C}$.

A curva apresentada na Figura 1 indica que o ajuste dos modelos e a obtenção dos coeficientes tornam-se mais complexos em razão da ocorrência da nitrificação, que, nesse caso, foi observada por volta do quinto dia de incubação das amostras. Em amostras incubadas sob temperatura padrão de análise $\left(20^{\circ} \mathrm{C}\right)$, considerou-se que a demanda nitrogenada seria expressiva no oitavo dia de incubação, havendo a expectativa de que fosse encontrado um pico após esse dia. A consideração de dados nesses períodos pode causar ajustes ruins dos modelos de primeira ordem, que possuem menores valores esperados de $\mathrm{DBO}_{u}$

Plotando-se os valores de DBO exercida no gráfico e utilizando-se regressão não linear para o ajuste da equação de primeira ordem (Equação 1), foram obtidos os valores de $\mathrm{k} \mathrm{e} \mathrm{DBO}_{\mathrm{u}}$ apresentados na Tabela 2.

Como esperado, verificou-se aumento na taxa de degradação do material orgânico com a elevação da temperatura. Utilizando-se a Equação 3, foi obtido valor de $\theta$ igual a 1,024, o qual difere dos valores de Streeter \& Phelps (1925), que são de 1,047, quando esses autores analisaram efluentes sanitários de baixa concentração, e de Oliveira et al. (2005), que observaram valor de 1,019 para esgoto bruto. Apesar da diferença, esses trabalhos e outros convergem ao concluir que há aumento na taxa de degradação do material orgânico das águas residuárias analisadas, não havendo consenso, porém, sobre o modelo que melhor descreveria a progressão da demanda bioquímica

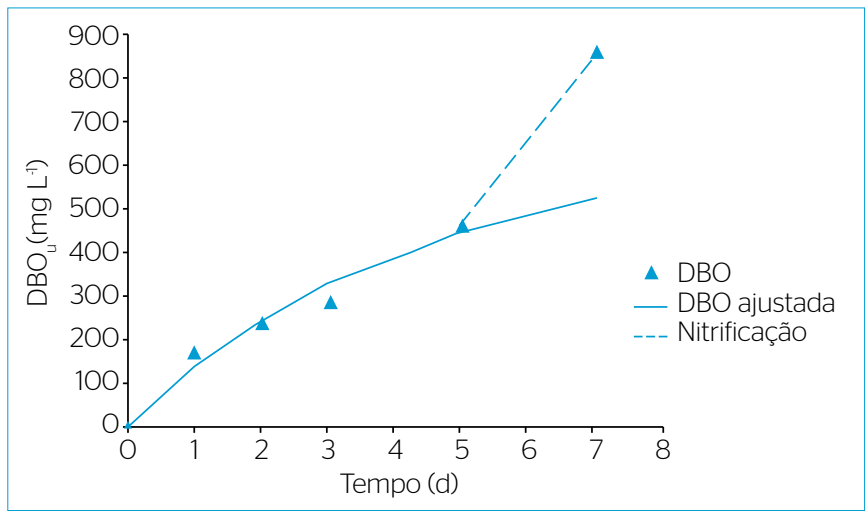

Gráfico 1 - Curva de progressão da demanda bioquímica de oxigênio carbonácea do esgoto doméstico bruto, incubado sob temperatura de $35^{\circ} \mathrm{C}$, obtida considerando dados até o quinto dia, com identificação de indícios da nitrificação na amostra. 
carbonácea e sobre a hipótese de a $\mathrm{DBO}_{\mathrm{u}}$ variar com a temperatura de incubação das amostras.

Para verificar se o modelo de segunda ordem descreve melhor a progressão de $\mathrm{DBO}$ do que a equação de primeira ordem, tal como afirmado por Adrian \& Sanders (1998), o modelo da Equação 2 foi ajustado aos dados obtidos experimentalmente, estando os valores apresentados na Tabela 3 .

Tal como reportado por Adrian \& Sanders (1998), a $\mathrm{DBO}_{\mathrm{u}}$ encontrada é maior no modelo de segunda ordem do que no modelo de primeira ordem. Já o valor de $\theta$ estimado para o EDB utilizado neste experimento - e utilizando-se os parâmetros obtidos após o ajuste da equação de reação de segunda ordem - foi de 1,057, valor próximo ao de 1,056, obtido por Schroepfer, Rosins e Susag (1964), citados por Khanh et al. (2011), em incubação das amostras sob temperatura na faixa de 20 a $30^{\circ} \mathrm{C}$. $\mathrm{O}$ valor de $\mathrm{k}$, obtido por meio do produto entre o $\mathrm{k}$ " e a $\mathrm{DBO}_{\mathrm{u}}$, apesar de ter a mesma unidade que o $\mathrm{k}$, é apresentado apenas a título de comparação com aquele obtido após o ajuste dos dados ao modelo de primeira ordem. Esse coeficiente foi obtido com o intuito único de possibilitar comparações, não sendo, no entanto, recomendado o seu emprego na Equação 1, já que trata-se de um parâmetro secundário e obtido a partir de outras duas estimativas.

Os dois modelos apresentaram elevado $\mathrm{R}^{2}$, sendo de 0,9848 e 0,9731 , respectivamente, para as temperaturas de 20 e $35^{\circ} \mathrm{C}$ com ajuste ao modelo de primeira ordem; e de 0,9866 e 0,9757, com ajuste ao modelo de segunda ordem. Avaliando-se o valor de $\mathrm{R}^{2}$, há de se considerar que ambos os modelos descreveram bem a progressão da DBO exercida, tanto na incubação sob temperatura padrão $\left(20^{\circ} \mathrm{C}\right)$ como na de $35^{\circ} \mathrm{C}$. Os valores de RMSE e AIC estão apresentados na Tabela 4.

Tabela 2 - Coeficientes de desoxigenação e demandas bioquímicas de oxigênio últimas em amostras de esgoto doméstico incubadas sob temperaturas de 20 e $35^{\circ} \mathrm{C}$, ajustados na equação de reação de primeira ordem.

\begin{tabular}{c|c|c|c}
\hline Temperatura $\left.{ }^{\circ} \mathrm{C}\right)$ & $\mathrm{k}\left(\mathrm{d}^{-1}\right)$ & $\mathrm{DBO}_{\mathrm{u}}\left(\mathrm{mg} \mathrm{L}^{-1}\right)$ & $\mathrm{R}^{2}$ \\
\hline 20 & 0,1593 & 579 & 0,9848 \\
\hline 35 & 0,2262 & 661 & 0,9731 \\
\hline
\end{tabular}

k: coeficiente de desoxigenação de primeira ordem; $\mathrm{DBO}_{\cup}$ : demanda bioquímica última; $\mathrm{R}^{2}$ : coeficiente de determinação.

Tabela 3 - Coeficientes de desoxigenação de primeira e segunda ordens e demandas últimas de oxigênio obtidos com a incubação de amostras sob temperaturas de 20 e $35^{\circ} \mathrm{C}$, ajustados na equação de segunda ordem.

\begin{tabular}{c|c|c|c|c}
\hline Temperatura $\left({ }^{\circ} \mathrm{C}\right)$ & $\mathrm{k}^{\prime \prime}\left(\mathrm{L} \mathrm{d}^{-1} \mathrm{mg}^{-1}\right) \times 10^{-4}$ & $\mathrm{DBO}_{\mathrm{u}}\left(\mathrm{mg} \mathrm{L}^{-1}\right)$ & $\mathrm{k}^{\prime}\left(\mathrm{d}^{-1}\right)$ & $\mathrm{R}^{2}$ \\
\hline 20 & 1,2864 & 878 & 0,1129 & 0,9866 \\
\hline 35 & 1,5803 & 1.006 & 0,1590 & 0,9757 \\
\hline
\end{tabular}

k": coeficiente de desoxigenação de segunda ordem; $\mathrm{DBO}_{\text {: }}$ demanda bioquímica última; ': coeficiente, de mesma unidade do coeficiente de desoxigenação de primeira ordem, obtido por meio do produto entre o coeficiente de segunda ordem e a demanda bioquímica última ( $\mathrm{k}^{\prime}=\mathrm{k}^{\prime \prime}$. DBO $\mathrm{DB}_{\mathrm{u}}$; $\mathrm{R}^{2}$ : coeficiente de determinação.
Embora esses critérios devam ser considerados com ressalvas, tanto o RMSE como o AIC indicaram que o modelo de segunda ordem foi ligeiramente melhor, ainda que essas diferenças sejam pouco importantes. Assim, com base nas diferenças ínfimas encontradas nos valores apresentados nas Tabelas 3 e 4, conclui-se que ambos os modelos podem descrever a progressão da DBO exercida. Considerando-se, no entanto, a simplicidade, o maior uso e conhecimento do modelo de decaimento de primeira ordem, recomenda-se a continuação do seu uso na predição da progressão da DBO exercida.

Marske \& Polkowski (1972) afirmaram que modelos de primeira ordem com $\mathrm{k}$ menor que $0,34 \mathrm{~d}^{-1}$ descrevem melhor a progressão da DBO exercida com o tempo. Neste trabalho, os modelos de primeira ordem apresentaram $\mathrm{k}$ de 0,1593 e $0,2262 \mathrm{~d}^{-1}$, valores que estão dentro da faixa de adequação da utilização de modelos de primeira ordem, segundo os citados autores, tendo sido, da mesma forma que os de segunda ordem, capazes de predizer a progressão da DBO exercida.

Os valores de $\mathrm{DBO}_{\mathrm{u}}$ estimados utilizando-se os dois diferentes modelos (primeira e segunda ordens), para temperaturas de 20 e $35^{\circ} \mathrm{C}$, foram substituídos na Equação 4, sendo obtidos os valores de $\varphi$ que estão apresentados na Tabela 5.

Os coeficientes obtidos foram maiores do que os encontrados nos trabalhos de Gotaas (1948), Zanoni (1967) e May (1971), com $\varphi$ iguais a 0,0013, 0,0033 e 0,0032, respectivamente, mas foram bem inferiores aos obtidos por Theriault (1927). O valor encontrado neste trabalho aproximou-se dos obtidos por Moore (1941), quando esse autor trabalhou com águas contendo pequena concentração de N. Como neste experimento houve emprego de dados numa etapa de menor desenvolvimento de bactérias nitrificantes, e com expurgo de dados tardios na progressão da DBO exercida na incubação das amostras

Tabela 4 - Erros padrão de estimativa e critério de informação de Akaike dos dados estimados usando modelos de primeira e segunda ordens para progressões da demanda bioquímica de oxigênio exercida, obtidos sob temperaturas de 20 e $35^{\circ} \mathrm{C}$ na incubação das amostras.

\begin{tabular}{l|c|c|c|c}
\multirow{2}{*}{ Parâmetros } & \multicolumn{2}{|c|}{$20^{\circ} \mathrm{C}$} & \multicolumn{2}{c}{$35^{\circ} \mathrm{C}$} \\
\cline { 2 - 5 } & $\begin{array}{c}\text { Primeira } \\
\text { ordem }\end{array}$ & $\begin{array}{c}\text { Segunda } \\
\text { ordem }\end{array}$ & $\begin{array}{c}\text { Primeira } \\
\text { ordem }\end{array}$ & $\begin{array}{c}\text { Segunda } \\
\text { ordem }\end{array}$ \\
\hline RMSE & 22,86 & 21,45 & 31,91 & 30,31 \\
\hline AIC & 53,12 & 52,36 & 62,08 & 61,56 \\
\hline
\end{tabular}

RMSE: raiz quadrada do erro quadrático médio; AIC: critério de informação de Akaike.

Tabela 5 - Coeficientes de temperatura da demanda última obtidos após o ajuste dos modelos de primeira e segunda ordens.

\begin{tabular}{l|c|c|c} 
Modelos & $\mathrm{DBO}_{u}^{20}$ & $\mathrm{DBO}_{u}^{35}$ & $\varphi$ \\
\hline Primeira ordem & 579 & 661 & 0,0095 \\
\hline Segunda ordem & 878 & 1.006 & 0,0098 \\
\hline
\end{tabular}

$\mathrm{DBO}_{U}^{20}$ : demanda bioquímica última a $20^{\circ} \mathrm{C}$; $\mathrm{DBO}_{U}^{35}$ : demanda bioquímica última a $35^{\circ} \mathrm{C}$ : $\varphi$ : coeficiente de temperatura da demanda bioquímica última. 
sob temperatura de $35^{\circ} \mathrm{C}$, considerou-se eliminada a influência da nitrificação no processo. Assim, apesar da maior concentração de $\mathrm{N}$ no EDB utilizado neste experimento, em relação ao conduzido por Moore (1941) e ao valor típico de $45 \mathrm{mg} \mathrm{L}^{-1}$ (VON SPERLING, 2014), acredita-se que não houve tempo de incubação suficiente para dar início ao processo de nitrificação.

A diferença entre os valores de $\mathrm{DBO}_{\mathrm{u}}^{20}$ e $\mathrm{DBO}_{\mathrm{u}}^{35}$, obtidos no ajuste da equação de primeira ordem, foi de 12,45\%. Quando eles foram obtidos a partir do ajuste da equação de segunda ordem, a diferença foi de $12,77 \%$. É importante ressaltar que essas diferenças podem ser consideradas pequenas, já que estão dentro da faixa de variação comum aos valores de $\mathrm{DBO}$, não sendo adequado atribuir conclusivamente à temperatura uma influência significativa e direta nos valores da $\mathrm{DBO}_{\mathrm{u}}$.

\section{CONCLUSÕES}

- Os modelos de primeira e segunda ordens descreveram bem a progressão da DBO exercida, tanto para a incubação das amostras de esgoto doméstico sob temperatura padrão $\left(20^{\circ} \mathrm{C}\right)$ como sob temperatura de $35^{\circ} \mathrm{C}$;

- Verificou-se, a partir da análise dos dados obtidos, que não se pode atribuir à temperatura influência na demanda bioquímica última $\left(\mathrm{DBO}_{\mathrm{u}}\right)$, já que as pequenas diferenças encontradas podem ser atribuídas à variabilidade normal na determinação da DBO exercida, que é intrínseca ao método de Winkler;

- Em vista da não confirmação da influência da temperatura de incubação das amostras na progressão da DBO exercida e nos valores de $\mathrm{DBO}_{\mathrm{u}}$, considera-se aceitável a estimativa da $\mathrm{DBO}$ exercida em amostras de esgoto doméstico incubadas a $35^{\circ} \mathrm{C}$, em substituição aos valores que seriam obtidos sob a temperatura padrão $\left(20^{\circ} \mathrm{C}\right)$.

\section{AGRADECIMENTOS}

Ao Conselho Nacional de Desenvolvimento Científico e Tecnológico $(\mathrm{CNPq})$, pelo apoio à realização do estudo.

\section{REFERÊNCIAS}

ADRIAN, D.D. \& SANDERS, T.G. (1998) Oxygen sag equation for second-order BOD decay. Water Research, v. 32, n. 3, p. 840-848.

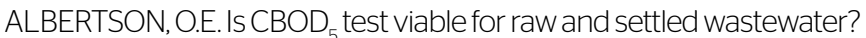
Journal of Environmental Engineering, v. 121, n. 7. p. 515-520, 1995.

APHA. American Public Health Association; AWWA. American Water Works Association; WEF. Water Environment Federation. (2012) Standard methods for the examination of water and wastewater. 22. ed. Washington: APHA/AWWA/WEF.

BORSUK, M.E. \& STOW, C.A. (2000) Bayesian parameter estimation in a mixed-order model of BOD decay. Water Research, v. 34, n. 6, p. 1830-1836.

COURCHAINE, R.J. (1968) Significance of nitrification in stream analysis: effects on the oxygen balance. Journal (Water Pollution Control Federation), v. 40, n. 5. p. 835-847.

DAGUE, R.E. (1981) Inhibition of nitrogenous BOD and treatment plant performance evaluation. Journal (Water Pollution Control Federation), v. 53, n. 12, p. 1738-1741.

EMILIANO, P.C.; VIVANCO, M.J.; MENEZES, F.S.; AVELAR, F.G. (2009) Foundations and comparison of information criteria: Akaike and Bayesian. Revista Brasileira de Biometria, v. 27, p. 394-411.

GAGLIASSO, D.; HUMMEL, S.; TEMESGEN, H. (2014) A comparison of selected parametric and non-parametric imputation methods for estimating forest biomass and basal area. Open Journal of Forestry, v. 4, n. 1, p. 42-48.
GOTAAS, H.B. (1948) Effect of temperature on biochemica oxidation of sewage. Sewage Works Journal, v. 20, n. 3, p. 441-477.

GREEN, B. \& WARD, G.H. (2011) Ultimate biochemical oxygen demand in semi-intensively managed shrimp pond waters. Aquaculture, n. 319, p. 253-261.

ISOLDI, L.A.; KOETZ,P.R.; IOLDI, L.A.(2005) Pós-tratamento de efluente nitrificado da parboilização de arroz utilizando desnitrificação em reator UASB. Engenharia Sanitária e Ambiental, v. 10, n. 4, p. 271-277.

JI, Y.; WANG, Y.; SUN, J.; YAN, T.; LI, J.; ZHAO, T., YIN, X.; SUN, C. (2O10) Enhancement of biological treatment of wastewater by magnetic field. Bioresource Technology, v. 101, n. 20, p. 8535-8540.

KHANH, D.; QUAN, L.; ZHANG, W.; HIRA, D.; FURUKAWA, K. (2O11) Effect of temperature on low-strength wastewater treatment by UASB reactor using poly(vinyl alcohol)-gel carrier. Bioresource Technology, v. 102, n. 24, p. 11147-11154.

MARSKE, D.M. \& POLKOWSKI, L.B. (1972) Evaluation of methods for estimating biochemical oxygen demand parameters. Journal (Water Pollution Control Federation), v. 44, n. 10, p. 1987-2000.

MASON, I.G.; MCLACHLAN, R.I.; GÉRARD, D.T. (2006) A double exponential model for biochemical oxygen demand. Bioresource Technology, v. 97, n. 2, p. 273-282.

MATOS, A.T. (2012) Qualidade do meio físico: práticas de laboratório. Viçosa: Imprensa Universitária, UFV. 150 p. 
MATOS, M.P.; BORGES, A.C.; MATOS, A.T.; SILVA, E.F.; MARTINEZ, M.A. (2014) Effect of time-temperature binomial in obtaining biochemical oxygen demand of different wastewaters. Engenharia Agrícola, v. 34, n. 2, p. 332-340.

MAY, T.G. (1971) The effect of temperature on the ultimate BOD of sewage. Science Thesis (Mastering in Civil Engineering) Northwestern University, Evanston, Illinois.

MOORE, E.W. (1941) Long-time biochemical oxygen demands at low temperatures. Sewage Works Journal, v. 13, n. 3. p. 561-577.

MUIRHEAD, W.M.; FARMER, G.; WALKER, S.; ROBB, L.; ELMENDORF, H.; MATTHEWS, R.; BUTLER, R.; MELCER, H. (2006) Study of raw wastewater $\mathrm{BOD}_{5}$ and $\mathrm{CBOD}_{5}$ relationship yields surprising results. WEFTEC, p. 840-853.

OKE, I.A. \& AKINDAHUNSI, A.A. (2005) A statistical evaluation of methods of determining BOD rate. Journal of Applied Sciences Research, v. 1, n. 2, p. 223-227.

OLIVEIRA, E.C.A.; ARAÚJO, G.M.; ARAÚJO, A.L.C.; BEDA, J.N.; INGUNZA, M.P.D. (2005) Caracterização e verificação das variações das taxas de degradação de matéria orgânica em diferentes temperaturas de incubação na ETE Ponta Negra/RN. In: Congresso Brasileiro de Engenharia Sanitária e Ambiental, 23., Campo Grande. Anais... Campo Grande, MS: ABES, 2005.
SILVA, E.F.; BORGES, A.C.; ANDRADE, F.H.B.; MATOS, A.T; MATOS, M.P. (2013) Desempenho de um sistema fossa biodigestora/alagado construído no tratamento de esgotos de residência rural. In: Simpósio Brasileiro sobre Aplicação de Wetlands Construidos no Tratamento de Águas Residuárias, 1., Florianópolis. Anais... Florianópolis, SC, 2013.

SIMON, F.X:; PENRU, Y; GUASTALLI, A.R;; LLORENS, J; BAIG, S. (2O11) Improvement of the analysis of the biochemical oxygen demand (BOD) of Mediterranean seawater by seeding control. Talanta, v. 85, n. 1, p. 527-532.

STREETER, H.W. \& PHELPS, E.B. (1925) A study of the natural purification of the Ohio River. Public Health Bulletin 146. Washington, D.C.: Public Health Service.

TCHOBANOGLOUS, G. \& EDDY, M. (2003) Wastewater engineering: treatment disposal and reuse. 4. ed. New York: McGraw-Hill. 1818p.

THERIAULT, E.J. (1927) The oxygen demand of polluted waters. Public Health Bulletin 173. Washington, D.C.: Public Health Service.

VON SPERLING, M. (2014) Introdução à qualidade das águas e ao tratamento de esgotos: princípios do tratamento biológico de águas residuárias. 4. ed. Belo Horizonte, MG: Editora da UFMG. v. 1. 470p.

ZANONI, A.E. (1965) Use for frozen wastewater as a test substrate. Public Works, n. 96, p. 72-74.

ZANONI, A.E. (1967) Waste water deoxygenation at different temperatures. Water Research, v. 1, p. 543-566. 